\title{
The Schwinger variational principles for one-dimensional quantum scattering
}

\author{
Dedicated to the Memory of Hermann Weyl
}

By

\section{L. DOLPH and R. K. RITT}

\section{§1. Introduction}

The success of variational methods in the solution of scattering problems [9] has aroused an interest in the rigorous formulation and deeper understanding of these methods $[1],[4],[6]$. In this paper, we examine the variational principle for the one-dimensional quantum scattering of a plane wave with positive energy, $k^{2}$, by a positive potential, $V(x)$, for which $\int_{-\infty}^{\infty} V(x) d x<\infty$.
We find:

1. If $\int_{-\infty}^{\infty} V(x) d x<2 k$, the Schwinger variational principle is valid; i.e., the behavior of the transmitted wave for a large $x$ can be determined by the stationary value of an appropriate bilinear integral form, subject to a normalizing constraint. If $\int_{-\infty}^{\infty} V(x) d x \geq 2 k$ an additional hypothesis must be imposed before the validity of the principle can be established; namely, the potential must not be "perfectly transparent" to the plane wave. To the best of our knowledge, this condition does not appear explicitly in the literature, although it is tacitly present in all the discussions of the principle. We call attention to an elementary example to show that a perfectly transparent potential can occur.

2. It is known [7] that in the one-dimensional case both the transmission and reflection coefficients can be characterized formally as stationary points of a variational principle. In the case of the reflection coefficient, the variational principle is symmetric with respect to a real inner product or, since it is convenient to use the theory of complex Hilbert space, quadratic with respect to a Hermitian inner product. In the case of the transmission coefficient, the variational principle is bilinear with respect to either inner product. However, as we will demonstrate, it can be reformulated as a quadratic variational principle in a suitably defined product Hilbert space. This reformulation not only makes a geometrical interpretation possible but in addition it results in unifying the theory since, when it is considered abstractly, it includes the variational principle for the reflection amplitude as a special case. 
It should be noticed that our formulation differs from the usual one, in which the competing functions do not lie in a Hilbert space since they do not vanish at infinity. However, by multiplying the integral equation by $U(x)=[V(x)]^{\frac{1}{4}}$ if $V(x) \geq 0$, and by $U_{1}(x)=[|i V(x)|]^{\frac{1}{2}}$ if $V(x) \leqq 0$, this formulation can be restated in terms of a Hilbert space. The first of these devices has been used by Kato [5]. Throughout this paper we will assume $V(x) \geqq 0$ and work with $U(x)$. It is a simple matter for the reader to verify that the results and method of proof are similar under the alternate hypothesis.

3. In the case of the transmission coefficient, the stationary points form a one (complex) parameter curve on the constraining surface. These points can be divided into two classes, according to the behavior of the imaginary part of the quadratic form. At points of the first class, the imaginary part exhibits a local saddle point behavior; i.e., we express this imaginary part as a real quadratic form on the direct sum of the two real Hilbert spaces, and show that its projections onto these two spaces have, respectively, a local minimum and a local maximum. At points of the second class, one of these two projections has a global maximum and nothing can be said about the other.

In the case of the reflection coefficient, the situation is much simpler geometrically. There is only one class of possible stationary points and it forms a one-dimensional linear manifold. Here the projections of the imaginary part of the corresponding real quadratic form on the direct sum of the two corresponding real Hilbert spaces always leads to a global minimum and a global maximum so that there is always a global saddle point behavior. On the other hand, in this case we obtain less information concerning the reflection coefficient itself, since there appears to be no useful analog of the crosssection theorem from which to deduce its properties.

4. For the sake of clarity and at the expense of some unity in the treatment we first discuss the more difficult case of the transmission coefficient, reserving the treatment of the reflection coefficient for the last section of the paper. The outline of the paper is as follows: $\S 2$ is devoted to a discussion of the pertinent properties of the associated Fredholm integral equations which underlie our entire theory; $\S 3$ is devoted to a discussion to the relation of these equations with the scattering problem and to an example of a "transparent" potential; $\S 4$ discusses the bilinear variational principle for the transmission coefficient in Hilbert space; $\S 5$ discusses the quadratic formulation of the same principle in a product Hilbert space; $\S 6$ is devoted to the characterization of the stationary points for the transmission coefficient; and $\S 7$ is given over to the corresponding formulation and discussion appropriate for the reflection coefficient.

The notation will, for the most part, be self-explanatory. The symbol $(*, *)$ will always be used to denote the inner product, regardless of the space with which we are dealing; the objects which appear in place of the asterisks will identify the space. We freely use the $\delta$ of the calculus of variations; 
this is equivalent to using Gateaux differentials [3]. When we say that a function is stationary we mean that its Gateaux differential is zero. The reader may be troubled by the extensive use of conjugations: in some cases the ordinary conjugation in a complex function space; in others a more abstract conjugation [8]. This could be avoided by using a symmetric, rather than a Hermitian symmetric, inner product, but this would involve a reorientation for the reader familiar with Hilbert spaces; we have chosen what appears to be the lesser of two evils.

The argument does not depend upon the existence of a biorthogonal expansion. It can be proved, using methods of Dunford, Schwartz, Werner, and Bade [2], that the basic integral operator does have a biorthogonal spectral resolution. Thus, there is a possibility that the problem could be formulated analogously to the Rayleigh-Ritz procedure [1].

\section{§2. The Fredholm equations}

We shall consider the operator on $L^{(2)}(-\infty, \infty)$ defined by

$$
K \varphi=\frac{1}{2 k i} \int_{-\infty}^{\infty} U(x) e^{i k|x-y|} U(y) \varphi(y) d y
$$

where $U(x)$ is a real valued function in $L^{(2)}(-\infty, \infty)$.

Lemma $1 . K$ is a bounded linear operator. The operator norm of $K,\|\mid K\|$, satisfies

$$
\|K\| \leq \frac{1}{2 k}\|U(x)\|^{2} .
$$

$K$ is compact. The adjoint of $K, K^{*}$ satisfies

$$
K^{*} \varphi=\overline{K \bar{\varphi}} \text {. }
$$

If $\varphi_{0}$ is defined by

$$
\varphi_{0}(x)=U(x) e^{i k x},
$$

then

$$
(K \varphi, \psi)-(\varphi, K \psi)=\frac{1}{2 k i}\left\{\left(\varphi, \varphi_{0}\right)\left(\varphi_{0}, \psi\right)+\left(\varphi, \bar{\varphi}_{0}\right)\left(\bar{\varphi}_{0}, \psi\right)\right\},
$$

for all $\varphi$ and $\psi$ in $L^{(2)}(-\infty, \infty)$.

Proof. (2.2) is obtained from Schwartz's inequality. $K$ is generated by a Hilbert-Schmidt kernel and is therefore compact. (2.3) is obtained from inspection. To prove (2.5) we integrate by parts as follows:

$$
\begin{aligned}
(K \varphi, \psi)= & \frac{1}{2 k i} \int_{-\infty}^{\infty} \overline{\psi(x)} \int_{-\infty}^{\infty} U(x) e^{i k|x-y|} U(y) \varphi(y) d y d x \\
= & \frac{1}{2 k i} \int_{-\infty}^{\infty} U(x) \overline{\psi(x)}\left\{e^{i k x} \int_{-\infty}^{x} e^{-i k y} U(y) \varphi(y) d y+\right. \\
& \left.+e^{-i k x} \int_{x}^{\infty} e^{i k y} U(y) \varphi(y) d y\right\} d x
\end{aligned}
$$




$$
\begin{aligned}
& =\frac{1}{2 k i}\left[\int_{-\infty}^{x} U(y) \overline{\psi(y)} e^{i k y} d y \int_{-\infty}^{x} e^{-i k y} U(y) \varphi(y) d y\right]_{-\infty}^{\infty}- \\
& \quad-\frac{1}{2 k i}\left[\int_{x}^{\infty} U(y) \overline{\psi(y)} e^{-i k y} d y \int_{x}^{\infty} e^{i k y} U(y) \varphi(y) d y\right]_{-\infty}^{\infty}- \\
& \quad-\frac{1}{2 k i} \int_{-\infty}^{\infty}\left(\int_{-\infty}^{x} U(y) \overline{\psi(y)} e^{i k y} d y\right) e^{-i k x} U(x) \varphi(x) d x- \\
& \quad-\frac{1}{2 k i} \int_{-\infty}^{\infty}\left(\int_{x}^{\infty} U(y) \overline{\psi(y)} e^{-i k y} d y\right) e^{i k x} U(x) \varphi(x) d x \\
& =\frac{1}{2 k i}\left\{\left(\varphi, \varphi_{0}\right)\left(\varphi_{0}, \psi\right)+\left(\varphi, \varphi_{0}\right)\left(\varphi_{0}, \psi\right)\right\}+ \\
& \quad+\int_{-\infty}^{\infty} \varphi(x)\left(\frac{1}{2 k i} \int_{-\infty}^{\infty} U(x) e^{-i k|x-y|} U(y) \psi(y) d y\right) d x \\
& =\frac{1}{2 k i}\left\{\left(\varphi, \varphi_{0}\right)\left(\varphi_{0}, \psi\right)+\left(\varphi, \bar{\varphi}_{0}\right)\left(\bar{\varphi}_{0}, \psi\right)\right\}+(\varphi, K \psi) .
\end{aligned}
$$

Lemma 2. The imaginary part of every eigenvalue of $K$ is non-positive, and the eigenfunction $\varphi$ corresponds to a real eigenvalue if and only if $\left(\varphi, \varphi_{0}\right)=$ $\left(\varphi, \bar{\varphi}_{0}\right)=0$.

Proof. If $K \varphi=\lambda \varphi$, then from (2.5),

$$
(\lambda-\bar{\lambda})\|\varphi\|^{2}=\frac{1}{2 k i}\left\{\left|\left(\varphi, \varphi_{0}\right)\right|^{2}+\left|\left(\varphi, \bar{\varphi}_{0}\right)\right|^{2}\right\} .
$$

Now, we define the operators

$$
F=\frac{K-K^{*}}{2 i}, \quad G=\frac{K+K^{*}}{2} .
$$

Lemma 3. $F$ and $G$ are bounded, self-adjoint, compact linear operators. $K=G+i F . F$ is negative semi-definite. The range of $F$ is the two dimensional space, $H_{0}$, spanned by $\varphi_{0}$ and $\bar{\varphi}_{0}$.

Proof. The first two statements are trivial. Using (2.5) and (2.6) we have

$$
F \varphi=-\frac{1}{4 k}\left\{\left(\varphi, \varphi_{0}\right) \varphi_{0}+\left(\varphi, \bar{\varphi}_{0}\right) \bar{\varphi}_{0}\right\},
$$

which proves the lemma.

Now, suppose $K \varphi=\varphi$. Then, from lemma 2 and (2.7), $F \varphi=0$. Therefore, $G \varphi=K \varphi=\varphi$. Conversely, if $G \varphi=\varphi$ and $F \varphi=0, K \varphi=\varphi$.

LEMMA 4. Let $H_{1}$ be the intersection of the null space of $I-G(I=$ identity $)$ and $H_{0}^{\perp}$. Then $H_{1}$ is the null space of $A=I-K$, and $H=H_{1}^{\perp}$ remains invariant under $K . H_{1}$ is also the null space for $A^{*}=I-K^{*}$, and $H$ remains invariant under $K^{*}$.

Proof. The paragraph which precedes the lemma shows that $A \varphi=0$ if and only if $F \varphi=0$ and $G \varphi=\varphi$; this is equivalent to $F^{*} \varphi=0$ and $G^{*} \varphi=\varphi$; 
thus, $H_{1}$ is also the null space for $A^{*}$. Let $\psi$ be in $H$. Then $(\psi, \varphi)=0$ for all $\varphi$ in $H_{1}$. But $(K \psi, \varphi)=\left(\psi, K^{*} \varphi\right)=\left(\psi, G^{*} \varphi\right)=0$, for all $\varphi$ in $H_{1}$. Therefore $K \psi$ is in $H$. Similarly, $K^{*} \psi$ is in $H$. This completes the proof.

THEOREM 1. The operators $A=I-K, A^{*}=I-K^{*}$, when regarded as operators on $H$ both have bounded inverses. In particular, the Fredholm equations

$$
A \varphi=\varphi_{0} ; \quad A^{*} \bar{\psi}=\varphi_{0},
$$

have unique solutions in $H$.

Proof. This follows directly from lemma 4. It should be observed that if the inhomogeneous terms in (2.8) are replaced by any functions in $H$, the theorem continues to be true. We use $\bar{\psi}$.rather than $\psi$ in (2.8) for later convenience.

THEOREM 2. If $\varphi_{1}$ and $\bar{\psi}_{1}$ are the solutions of (2.8) then

$$
\left(\varphi_{1}, \varphi_{0}\right)=\left(\varphi_{0}, \bar{\psi}_{1}\right) \quad(\text { Reciprocity }) .
$$

If $\|U(x)\|^{2}<2 k$, then $\left(\varphi_{1}, \varphi_{0}\right) \neq 0$.

Proof. $\left(\varphi_{1}, \varphi_{0}\right)=\left(\varphi_{1}, A^{*} \bar{\psi}_{1}\right)=\left(A \varphi_{1}, \bar{\psi}_{1}\right)=\left(\varphi_{0}, \bar{\psi}_{1}\right)$; if $A \varphi_{1}=\varphi_{1}-K \varphi_{1}=\varphi_{0}$, and $\left(\varphi_{1}, \varphi_{0}\right)=0$, then $\left\|\varphi_{1}\right\|^{2}=\left(K \varphi_{1}, \varphi_{1}\right) \leqq\|K\|\left\|\varphi_{1}\right\|^{2}$. Therefore $\|K\| \| \geqq 1$. But if $\|U(x)\|^{2}<2 k$, this contradicts (2.2). The theorem is proved.

In what follows, regardless of the value of $\|U(x)\|$, we shall assume that $\varphi_{1}$ satisfies

$$
\left(\varphi_{1}, \varphi_{0}\right) \neq 0
$$

As we shall see in $\S 3$ this is equivalent to assuming that the potential is not perfectly transparent. Restricting ourselves to $H$ rather than studying the equations $(2.8)$ on all of $L^{(2)}(-\infty, \infty)$ is not essential, as we shall see in Theorem 4 below. We do so as a matter of elegance,. since otherwise we would have to keep in mind the finite dimensional ( $G$ is compact) subspace, $H_{1}$, and phrase our statements accordingly.

\section{§3. Discussion of the preceding formulations}

We shall now connect the Fredholm equations (2.8) with the problem of one-dimensional quantum scattering. The fundamental analytic object one has to deal with is the time independent Schrödinger equation

$$
\omega^{\prime \prime}+\left[k^{2}-V(x)\right] \omega=0,
$$

subject to the conditions

$$
\begin{gathered}
\lim _{x \rightarrow \infty}\left[\omega^{\prime}-i k \omega\right]=0, \\
\lim _{x \rightarrow-8}\left[\omega^{\prime}+i k \omega\right] e^{-i k x}=2 i k .
\end{gathered}
$$


Solving this problem is equivalent to solving the integral equation

$$
\omega(x)=e^{i k x}+\frac{1}{2 k i} \int_{-\infty}^{\infty} e^{i k|x-y|} V(y) \omega(y) d y .
$$

If we assume that $\int_{-\infty}^{\infty}|V(y)| d y<\infty$, this is a Fredholm equation of the second kind in the space of essentially bounded functions. Now, if $V(y) \geq 0$, we avoid the difficulty of dealing with a space which is not self-dual, by setting $U(x)=[V(x)]^{\frac{1}{2}}, \varphi(x)=U(x) \omega(x), \varphi_{0}(x)=U(x) e^{i k x}$, and multiplying (3.4) by $U(x)$. This gives the first equation in (2.8). It is clear that the solution, $\varphi_{1}$, of (2.8) determines the solution of (3.4), except on the set for which $U(x)=0$. On this set $\omega(x)$ can be determined from (3.1) and the usual requirements of continuity of $\omega(x)$ and $\omega^{\prime}(x)$.

The critical quantity we wish eventually to determine is

$$
\frac{1}{\lambda_{1}}=\int_{-\infty}^{\infty} \omega(x) V(x) e^{-i k x} d x
$$

where $\omega(x)$ is the solution of (3.4). $1+\frac{1}{2 k i \lambda_{1}}$ is the complex amplitude of the transmitted radiation. But $\frac{1}{\lambda_{1}}$ is exactly the same as $\left(\varphi_{1}, \varphi_{0}\right)$. Thus we lose nothing by dealing with (2.8) rather than (3.4).

Now what is the structure of the space $H_{1}$ ? To understand this we shall interpret $H_{1}$ from the point of view of equation (3.1). Suppose that $\varphi(x)$ simultaneously satisfies

$$
\begin{aligned}
\varphi & =K \varphi, \\
\left(\varphi, \varphi_{0}\right) & =\left(\varphi, \bar{\varphi}_{0}\right)=0 .
\end{aligned}
$$

Then the corresponding $\omega(x)$ satisfies

$$
\begin{gathered}
\omega(x)=\frac{1}{2 k i} \int_{-\infty}^{\infty} e^{i k|x-y|} V(y) \omega(y) d y, \\
\int_{-\infty}^{\infty} \omega(x) V(x) e^{i k x} d x=\int_{-\infty}^{\infty} \omega(x) V(x) e^{-i k x} d x=0 .
\end{gathered}
$$

But then we can write

$$
\begin{gathered}
\omega(x)=\frac{1}{2 k i}\left\{e^{i k z} \int_{-\infty}^{x} e^{-i k y} V(y) \omega(y) d y+e^{-i k x} \int_{-x}^{\infty} e^{i k y} V(y) \omega(y) d y\right\}, \\
\omega^{\prime}(x)=\frac{1}{2}\left\{e^{i k x} \int_{-\infty}^{x} e^{-i k y} V(y) \omega(y) d y-e^{-i k x} \int_{-x}^{\infty} e^{i k y} V(y) \omega(y) d y\right\}, \\
\omega^{\prime \prime}+\left[k^{2}-V(x)\right] \omega=0 .
\end{gathered}
$$


Combining (3.10) and (3.11), and using (3.9) we secure the following conditions which must be satisfied by $\omega(x)$ :

$$
\begin{aligned}
\lim _{x \rightarrow \infty}\left[i k \omega+\omega^{\prime}\right] & =0 ; & \lim _{x \rightarrow \infty}\left[i k \omega-\omega^{\prime}\right] & =0, \\
\lim _{x \rightarrow-\infty}\left[i k \omega+\omega^{\prime}\right] & =0 ; & \lim _{x \rightarrow-\infty}\left[i k \omega-\omega^{\prime}\right] & =0 .
\end{aligned}
$$

From (3.13) and (3.14) it is apparent that $\omega(x)$ and $\omega^{\prime}(x)$ must approach zero as $x$ approaches both $+\infty$ and $-\infty$. The $\omega(x)$ then correspond to certain bound states of the system; i.e., solutions of the Schrödinger equation which together with their derivatives vanish at $+\infty$ and $-\infty$. In limiting ourselves to $H$ we exclude from consideration those $\varphi(x)$ which correspond to these bound states. It is only when we do this that we guarantee the uniqueness of the solution of (2.8). There may well exist other bound states. These will correspond to those eigenfunctions of $K$ which have real eigenvalues. This is easily verified by making use of lemma 2 and repeating the above argument. It serves no useful purpose to exclude these other bound states.

The condition (2.10) is, as we shall see, forced upon us if we wish to find (3.5) using a variational principle. In theorem 2 we proved that if the potential is "sufficiently weak", i.e.,

$$
\int_{-\infty}^{\infty} V(x) d x<2 k,
$$

then (2.10) is automatically satisfied. But what can be said if (3.15) fails to hold? Let us suppose that $\left(\varphi_{1}, \varphi_{0}\right)=0$. Since $\varphi_{1}=\varphi_{0}+K \varphi_{1},\left(K \varphi_{1}, \varphi_{1}\right)=$ $\left(\varphi_{1}, \varphi_{1}\right)=\left(\varphi_{1}, K \varphi_{1}\right)$. Consequently, using $(2.5),\left(\varphi_{1}, \bar{\varphi}_{0}\right)=0$. Then the corresponding $\omega(x)$, which is a solution of (3.4), satisfies the conditions (3.9). As in the discussion which led to (3.13) and (3.14), we obtain

$$
\begin{gathered}
\lim _{x \rightarrow \infty}\left[i k \omega+\omega^{\prime}\right] e^{-i k x}=\lim _{x \rightarrow-\infty}\left[i k \omega+\omega^{\prime}\right] e^{-i k x}=2 i k, \\
\lim _{x \rightarrow \infty}\left[i k \omega-\omega^{\prime}\right]=\lim _{x \rightarrow-\infty}\left[i k \omega-\omega^{\prime}\right]=0 .
\end{gathered}
$$

Therefore, we have

$$
\lim _{x \rightarrow \infty} \omega e^{-i k x}=\lim _{x \rightarrow-\infty} \omega e^{-i k x}=1 .
$$

It is (3.18) which supplies a precise meaning to the assertion that $V(x)$ is "perfectly transparent" to the incoming plane wave $e^{i k x}$. For large positive values of $x$, no scattering will be detected, and for large negative values of $x$, no reflection will be detected. That this can happen, even in a simple type of situation, can be seen by letting $k=4 \pi$, and defining

$$
V(x)= \begin{cases}12 \pi^{2} & 0 \leq x \leq 1 \\ 0 & \text { otherwise }\end{cases}
$$


then the function

$$
\omega(x)= \begin{cases}\frac{3}{2} e^{2 \pi i x}-\frac{1}{2} e^{-2 \pi i x} & 0 \leqq x \leq 1 \\ e^{4 \pi i x} & \text { otherwise }\end{cases}
$$

is the solution of (3.4). In this case, the variational principle will not apply, as will become apparent in $\S 4$. It is an open question to find the general conditions on $V(x)$ which will guarantee (2.10). Trivially, (2.10) fails to be satisfied if and only if $\left(A^{-1} \varphi_{0}, \varphi_{0}\right)=0$, but it is not obvious how to extract from this a sharp criterion. For a square wave potential, the question is easy to settle, but we shall not trouble the reader with the details.

\section{§4. The variational principle for the transmission coefficient}

We shall consider the variational technique of finding $\left(\varphi_{1}, \varphi_{0}\right)$. Consider the bilinear form

$$
J=(A \varphi, \bar{\psi}) .
$$

Let us attempt to make $J$ stationary, subject to the normalizing constraint

$$
\left(\varphi, \varphi_{0}\right)\left(\varphi_{0}, \bar{\psi}\right)=1 .
$$

To do this, we introduce the Lagrange multiplier, $\lambda$, and set

$$
J_{\lambda}=J-\lambda\left(\varphi, \varphi_{0}\right)\left(\varphi_{0}, \bar{\psi}\right) .
$$

Then the first variation of $J_{\lambda}$ is given by

(4.4) $\left\{\begin{aligned} \delta J_{\lambda} & =\left(\delta \varphi, A^{*} \bar{\psi}\right)+(A \varphi, \delta \bar{\psi})-\lambda\left(\delta \varphi, \varphi_{0}\right)\left(\varphi_{0}, \bar{\psi}\right)-\lambda\left(\varphi, \varphi_{0}\right)\left(\varphi_{0}, \delta \bar{\psi}\right) \\ & =\left(\delta \varphi, A^{*} \bar{\psi}-\bar{\lambda}\left(\bar{\psi}, \varphi_{0}\right) \varphi_{0}\right)+\left(A \varphi-\lambda\left(\varphi, \varphi_{0}\right) \varphi_{0}, \delta \bar{\psi}\right) .\end{aligned}\right.$

Therefore $J_{\lambda}$ is stationary if and only if

$$
A \varphi=\lambda\left(\varphi, \varphi_{0}\right) \varphi_{0}, \quad A^{*} \bar{\psi}=\bar{\lambda}\left(\bar{\psi}, \varphi_{0}\right) \varphi_{0} .
$$

THEOREM 3 (Schwinger's variational principle). There exists exactly one value of $\lambda, \lambda_{1}$, such that $J_{\lambda}$ can be made stationary. $\lambda_{1}$ is equal to the value of $J$ when $J$ is made stationary subject to the constraint (4.2). Simultaneously, $\lambda_{1}=\frac{1}{\left(\varphi_{1}, \varphi_{0}\right)}$. The pair, $\varphi, \psi$ make $J$ stationary subject to (4.2) if and only if there exists an $\alpha \neq 0$ such that $\varphi=\alpha \lambda_{1} \varphi_{1}, \psi=\frac{1}{\alpha} \lambda_{1} \psi_{1}$.

Proof. Since we are assuming (2.10), let $\lambda=\frac{1}{\left(\varphi_{1}, \varphi_{0}\right)}=\frac{1}{\left(\varphi_{0}, \bar{\psi}_{1}\right)}$. The last equality follows from theorem 2 . Then the pair $\varphi_{1}, \psi_{1}$ satisfy (4.5). Thus, $J_{\lambda}$ can be made stationary. On the other hand, if $J_{\lambda}$ can be made stationary, there exists a pair, $\varphi, \psi$ such that (4.5) holds. From theorem $1, A$ and $A^{*}$ have bounded inverses, so $\lambda\left(\varphi, \varphi_{0}\right) \neq 0$ and $\bar{\lambda}\left(\bar{\psi}, \varphi_{0}\right) \neq 0$. Then it is an easy matter, from the uniqueness of the solutions of (2.8), to see that $\varphi=\lambda\left(\varphi, \varphi_{0}\right) \varphi_{1}$, $\psi=\lambda\left(\varphi_{0}, \bar{\psi}\right) \psi_{1}$. But then taking the inner product of the first of these with 
$\varphi_{0}$, we find that $\lambda\left(\varphi_{1}, \varphi_{0}\right)=1$. Thus $\lambda=\lambda_{1}$ is uniquely determined. If $\varphi$ and $\psi$ satisfy (4.2), from (4.5) we obtain $\lambda=(A \varphi, \bar{\psi})$, and from-above, we obtain $\varphi=\alpha \lambda_{1} \varphi_{1}, \psi=\frac{1}{a} \lambda_{1} \psi_{1}$, where $\alpha=\left(\varphi, \varphi_{0}\right)$. Finally, for every $\alpha \neq 0$, $\alpha \lambda_{1} \varphi_{1}, \frac{1}{a} \lambda_{1} \psi_{1}$ satisfy both (4.5) and (4.2). To emphasize the importance of (2.10), we state explicitly:

CoRollary. If $\left(\varphi_{1}, \varphi_{0}\right)=0$ there does not exist a $\lambda$ such that $J_{\lambda}$ can be made stationary, and consequently, $J$ cannot be made stationary subject to (4.2).

The next theorem shows that in the application of the variational principle it is not necessary to distinguish between $H$ and $L^{(2)}(-\infty, \infty)$. Our use of the space $H$ has been a convenience for the easier statement and proof of theorem 3 .

THEOREM 4. Let $\varphi$ and $\vec{\psi}$ be in $L^{(2)}(-\infty, \infty)$. Then $\varphi$ and $\bar{\psi}$ have unique erpresentations as $\varphi^{\prime}+\varphi^{\prime \prime}, \bar{\psi}^{\prime}+\bar{\psi}^{\prime \prime}$, respectively, where $\varphi^{\prime}$ and $\bar{\psi}^{\prime}$ are in $H_{1}, A, \varphi^{\prime \prime}$ and $\bar{\psi}^{\prime \prime}$ are in $H(A \varphi, \bar{\psi})=\left(A \varphi^{\prime \prime}, \bar{\psi}^{\prime \prime}\right)$, and $\left(\varphi, \varphi_{0}\right)\left(\varphi_{0}, \bar{\psi}\right)=\left(\varphi^{\prime \prime}, \varphi_{0}\right)\left(\varphi_{0}, \bar{\psi}^{\prime \prime}\right)$.

Proof. The proof follows easily from lemma 3. In the language of $\S 3$, in the application of the variational principle, the failure to factor out those functions which correspond to bound states has no effect on the computed quantities, although it destroys the uniqueness of the solutions to (2.8).

THEOREM 5. Let $\lambda_{1}=\mu_{1}+i \nu_{1}$, where $\mu_{1}$ and $\nu_{1}$ are real. Then

Proof.

$$
\nu_{1}=\frac{1}{4 k}\left[1+\frac{\left|\left(\varphi_{1}, \bar{\varphi}_{0}\right)\right|^{2}}{\left|\left(\varphi_{1}, \varphi_{0}\right)\right|^{2}}\right] .
$$

$$
2 i \nu_{1}=\frac{\left(\varphi_{0}, \varphi_{1}\right)-\left(\varphi_{1}, \varphi_{0}\right)}{\left|\left(\varphi_{1}, \varphi_{0}\right)\right|^{2}}=\frac{\left(A \varphi_{1}, \varphi_{1}\right)-\left(\varphi_{1}, A \varphi_{1}\right)}{\left|\left(\varphi_{1}, \varphi_{0}\right)\right|^{2}}=-\left\{\frac{\left(K \varphi_{1}, \varphi_{1}\right)-\left(\varphi_{1}, K \varphi_{1}\right)}{\left|\left(\varphi_{1}, \varphi_{0}\right)\right|^{2}}\right\} .
$$

Using this and (2.5) we obtain (4.10). This theorem is the one-dimensional counterpart of the total cross-section theorem [7], [9]. It shows that the imaginary part of $\lambda_{1}$ is determined by the ratio of the reflected energy to the transmitted energy. If one assumes conservation of particles, theorem 5 can be proved analogously to the proof given by Schiff in the three-dimensional case [L. I. Schiff, Proc. Theor. Physics 11, 288 (1954)]. The authors are indebted to Professor Schiff for this observation.

\section{§5. The quadratic formulation}

The variational principle stated in $\S 4$ is in terms of a bilinear form and a bilinear constraint. We shall now show that the principle can be formulated as a quadratic problem. This will facilitate a heuristic understanding of the principle, and make clear the geometry of the "saddle" nature of the stationary point.

We introduce the space

$$
\underline{H}=H \oplus H \text {. }
$$


The elements of $\underline{H}$ will be of the form

$$
\underline{\varphi}=[\varphi, \psi],
$$

where $\varphi$ and $\psi$ are in $H$. If $\underline{\varphi}^{\prime}=\left[\varphi^{\prime}, \psi^{\prime}\right]$ and $\underline{\varphi}^{\prime \prime}=\left[\varphi^{\prime \prime}, \psi^{\prime \prime}\right]$ are in $\underline{H}$, their inner product will be given by

$$
\left(\underline{\varphi}^{\prime}, \underline{\varphi}^{\prime \prime}\right)=\left(\varphi^{\prime}, \varphi^{\prime \prime}\right)+\left(\psi^{\prime}, \psi^{\prime \prime}\right) .
$$

We now state two lemmas. Their proofs are matters of routine computation, and hence will be omitted.

Lemma 5. Let $\underline{C}$ be a mapping of $\underline{H}$ into $\underline{H}$ defined by

$$
\underline{C} \underline{\varphi}=\underline{C}[\varphi, \psi]=[\bar{\psi}, \bar{\varphi}] .
$$

Then $\underline{C}^{2}=\underline{I}$ (identity), $(\underline{C} \underline{\varphi}, \underline{C} \underline{\varphi})=(\underline{\varphi}, \underline{\varphi}), \underline{C}(\lambda \underline{\varphi})=\bar{\lambda} \underline{C} \underline{\varphi} . \quad$ If $\underline{C} \underline{\varphi}=\underline{\varphi}$ and $\underline{C} \underline{\psi}=\underline{\psi}$, then $(\underline{\varphi}, \underline{\psi})=(\underline{\psi}, \underline{\varphi})$ and this inner product is real. If

$$
\underline{\varphi}_{0}=\left[\varphi_{0}, \bar{\varphi}_{0}\right], \quad \underline{\varphi}_{0}=\left[\varphi_{0},-\bar{\varphi}_{0}\right]
$$

Lemma 6. Let $\underline{L}$ be the mapping of $\underline{H}$ into $\underline{H}$ defined by

$$
\underline{C}_{\varphi_{0}}=\underline{\varphi}_{0}, \quad \underline{C} \underline{\psi}_{0}=-\underline{\psi}_{0} .
$$

$$
\underline{L} \underline{\varphi}=\underline{L}[\varphi, \psi]=[A \varphi, A \psi] .
$$

Then, $\underline{L}$ is a linear operator; $\underline{L}^{*}$ the conjugate of $\underline{L}$ is given by

$$
\underline{L} \underline{\varphi}=\left[A^{*} \varphi, A^{*} \psi\right] \text {, }
$$

and

$$
\underline{L} \underline{C}=\underline{C} \underline{L}
$$

Now we define

$$
\underline{J}=2(\underline{L} \underline{\varphi}, \underline{C} \underline{\varphi}) .
$$

We shall consider the problem of making $I$ stationary subject to the constraint,

$$
\left(\underline{\varphi}, \underline{\varphi}_{0}\right)^{2}-\left(\underline{\varphi}, \underline{\varphi}_{0}\right)^{2}=4
$$

As in $\S 4$, we introduce the Lagrange multiplier, $\lambda$, and set

$$
\underline{J}_{\lambda}=\underline{J}-\lambda\left\{\left(\underline{\varphi}, \underline{\varphi}_{0}\right)^{2}-\left(\underline{\varphi}, \underline{\psi}_{0}\right)^{2}\right\}
$$

Then,

$$
\left\{\begin{aligned}
\delta \underline{J}_{\lambda}= & 2(\underline{L} \underline{\varphi}, \underline{C} \delta \underline{\varphi})+2(\underline{L} \delta \underline{\varphi}, \underline{C} \underline{\varphi})- \\
& -2 \lambda\left\{\left(\underline{\varphi}, \underline{\varphi}_{0}\right)\left(\delta \underline{\varphi}, \underline{\varphi}_{0}\right)-\left(\underline{\varphi}, \underline{\psi}_{0}\right)\left(\delta \underline{\varphi}, \underline{\psi}_{0}\right)\right\} \\
= & 2\left(\delta \underline{\varphi}, 2 \underline{C} \underline{L} \varphi-\bar{\lambda}\left\{\left(\underline{\varphi}_{0}, \underline{\varphi}\right) \underline{\varphi}_{0}-\left(\underline{\psi}_{0}, \underline{\varphi}\right) \underline{\psi}_{0}\right\}\right)
\end{aligned}\right.
$$


This last equality makes use of (5.10) and lemma 5. Making use of lemma.5, we find that $J_{\lambda}$ is stationary if and only if

$$
2 \underline{L} \underline{\varphi}=\lambda\left\{\left(\underline{\varphi}, \underline{\varphi}_{0}\right) \underline{\varphi}_{0}+\left(\underline{\varphi}, \underline{\varphi}_{0}\right) \underline{\varphi}_{0}\right\}
$$

But

$$
\left(\underline{\varphi}, \underline{\varphi}_{0}\right)=\left(\varphi, \varphi_{0}\right)+\left(\psi, \bar{\varphi}_{0}\right) ; \quad\left(\underline{\varphi}, \underline{\varphi}_{0}\right)=\left(\varphi, \varphi_{0}\right)-\left(\psi, \bar{\varphi}_{0}\right) .
$$

Therefore

$$
\left(\underline{\varphi}, \underline{\varphi}_{0}\right) \underline{\varphi}_{0}+\left(\underline{\varphi}, \underline{\psi}_{0}\right) \underline{\psi}_{0}=\left[2\left(\varphi, \varphi_{0}\right) \varphi_{0}, 2\left(\psi, \bar{\varphi}_{0}\right) \bar{\varphi}_{0}\right] .
$$

Therefore, $\underline{J}_{\lambda}$ is stationary at $\underline{\varphi}=[\varphi, \psi]$ if and only if

$$
A \varphi=\lambda\left(\varphi, \varphi_{0}\right) \varphi_{0}, \quad A^{*} \bar{\psi}=\bar{\lambda}\left(\bar{\psi}, \varphi_{0}\right) \varphi_{0} .
$$

This is a consequence of (5.17), (5.15), lemma 6, and (2.3). We have proved the following theorem:

THEOREM 6. There is a unique value of $\lambda, \lambda_{1}$, such that $\underline{J}_{\lambda_{1}}$ can be made stationary. $\lambda_{1}$ is the stationary value of $J$ subject to the constraint (5.12). $\lambda_{1}$ is the same as in theorem 3. $\varphi_{1}=\left[\lambda_{1} \varphi_{1}, \lambda_{1} \psi_{1}\right]$ is a stationary point, and all other stationary points are of the form $\underline{\varphi}(\alpha)=\left[\alpha \lambda_{1} \varphi_{1}, \frac{1}{\alpha} \lambda_{1} \psi_{1}\right], \alpha \neq 0$.

A heuristic interpretation of theorem 6 is that we are finding that member of a family of quadric surfaces which is tangent to a fixed hyperboloid. The tangency takes place along a one (complex) parameter curve, $\underline{\varphi}(\alpha)$, in the surface (5.12). If we re-paramatrize the curve $\varphi(\alpha)$ by letting $\alpha=e^{\beta}$, we see that the curve $\varphi(\alpha)$ is the orbit of $\underline{\varphi}_{1}$ under the analytic group of operators $e^{\underline{B} \beta}$, where $\underline{B} \underline{\varphi}=\underline{B}[\varphi, \psi]=[\varphi,-\bar{\psi}][3]$.

\section{§6. The imaginary part of $\underline{J}$}

If $\underline{\varphi}$ is a stationary point, and if $\underline{\varphi}+\delta \underline{\varphi}$ is any point which lies on the surface (5.12), we define

$$
\left\{\begin{aligned}
\Delta \underline{J} & =2(\underline{L}(\underline{\varphi}+\delta \underline{\varphi}), \underline{C}(\underline{\varphi}+\delta \underline{\varphi}))-2(\underline{L} \underline{\varphi}, \underline{C} \underline{\varphi}) \\
& =4(\underline{L} \underline{\varphi}, \underline{C} \delta \underline{\varphi})+2(\underline{L} \delta \underline{\varphi}, \underline{C} \delta \underline{\varphi}) .
\end{aligned}\right.
$$

Since both $\underline{\varphi}$ and $\underline{\varphi}+\delta \underline{\varphi}$ satisfy (5.12), we have

$$
2\left(\left(\underline{\varphi}, \underline{\varphi}_{0}\right) \underline{\varphi}_{0}+\left(\underline{\varphi}, \underline{\psi}_{0}\right) \underline{\psi}_{0}, \underline{C} \delta \underline{\varphi}\right)+\left(\delta \underline{\varphi}, \underline{\varphi}_{0}\right)^{2}-\left(\delta \underline{\varphi}, \underline{\psi}_{0}\right)^{2}=0 .
$$

But at a stationary point (5.15) holds. Combining this with (6.1) and (6.2) we secure

$$
\Delta \underline{J}=2(\underline{L} \delta \underline{\varphi}, \underline{C} \delta \underline{\varphi})-\lambda_{1}\left\{\left(\delta \underline{\varphi}, \underline{\varphi}_{0}\right)^{2}-\left(\delta \underline{\varphi}, \underline{\psi}_{0}\right)^{2}\right\} .
$$

Lemma 7 . If $\underline{\varphi}$ is in $\underline{H}$, we define

$$
\underline{u}=\frac{1}{2}(\underline{\varphi}+\underline{C} \underline{\varphi}), \quad \underline{v}=\frac{1}{2 i}(\underline{\varphi}-\underline{C} \underline{\varphi}) .
$$


Then

Also

$$
\underline{\varphi}=\underline{u}+i \underline{v}, \quad \underline{C} \underline{u}=\underline{u}, \quad \underline{C} \underline{v}=\underline{v} .
$$

$$
\begin{array}{rlrl}
\frac{1}{2}\left(\underline{\varphi}_{0}+\underline{C} \underline{\varphi}_{0}\right) & =\underline{\varphi}_{0}, & \frac{1}{2 i}\left(\underline{\psi}_{0}-\underline{C} \underline{\varphi}_{0}\right)=-i \underline{\varphi}_{0}, \\
\frac{1}{2 i}\left(\underline{\varphi}_{0}-\underline{C} \underline{\varphi}_{0}\right)=0, & \frac{1}{2}\left(\underline{\psi}_{0}+\underline{C} \underline{\varphi}_{0}\right)=0 .
\end{array}
$$

We define

$$
\underline{u}_{0}=\underline{\varphi}_{0} ; \quad \underline{v}_{0}=-i \underline{\varphi}_{0} .
$$

Then

$$
\underline{C} \underline{u}_{0}=\underline{u}_{0}, \quad \underline{C}_{0}=\underline{v}_{0} .
$$

Proof. Direct computation, using lemma 5.

LemMa 8. Let

$$
\underline{G}=\frac{1}{2}\left(\underline{L}+\underline{L}^{*}\right), \quad \underline{F}=\frac{1}{2 i}\left(\underline{L}-\underline{L}^{*}\right) .
$$

Then, $\underline{L}=\underline{G}+i \underline{F}, \underline{G}$ and $\underline{F}$ are self-adjoint operators, and $\underline{F}$ is positive semidefinite. Both $\underline{G}$ and $\underline{F}$ commute with $\underline{C}$, and if $\underline{C} \underline{\varphi}=\underline{\varphi}, \underline{C} \underline{\psi}=\underline{\varphi}$, then $(\underline{F} \underline{\varphi}, \underline{\varphi})$ $=(\underline{\psi}, \underline{F} \underline{\varphi})=(\underline{\varphi}, \underline{F} \underline{\varphi})=(\underline{F} \underline{\psi}, \underline{\varphi})$, and this inner product is real. Similarly for $\underline{G}$.

Proof. The first two assertions are obvious. To prove that $\underline{F}$ is positive semi-definite, we let $\varphi=[\varphi, \psi]$. Then

$$
\left\{\begin{aligned}
(\underline{F} \underline{\varphi}, \underline{\varphi}) & =\frac{1}{2 i}(\underline{L} \underline{\varphi}, \underline{\varphi})-\frac{1}{2 i}\left(\underline{L}^{*} \underline{\varphi}, \underline{\varphi}\right) \\
& =\frac{1}{2 i}([A \varphi, A \psi],[\varphi, \psi])-\frac{1}{2 i}\left(\left[A^{*} \varphi, A^{*} \psi\right],[\varphi, \psi]\right) \\
& =\frac{1}{2 i}\left\{\left(\left(A-A^{*}\right) \varphi, \varphi\right)+\left(\left(A-A^{*}\right) \psi, \psi\right)\right\} \\
& =-\frac{1}{2 i}\left\{\left(\left(K-K^{*}\right) \varphi, \varphi\right)+\left(\left(K-K^{*}\right) \psi, \psi\right)\right\} \\
& =-\{(F \varphi, \varphi)+(F \psi, \psi)\} .
\end{aligned}\right.
$$

Therefore, from lemma 3, $(\underline{F} \underline{\varphi}, \underline{\varphi}) \geq 0$. Now

$$
\underline{C} \underline{F}=-\frac{1}{2 i}\left(\underline{C} \underline{L}-\underline{C} \underline{L}^{*}\right)=\frac{1}{2 i}\left(\underline{L} \underline{C}-\underline{L}^{*} \underline{C}\right)=\underline{F} \underline{C},
$$

from lemmas 5 and 6 . Similarly $\underline{C} \underline{G}=\underline{G} \underline{C}$. Finally,

$$
(\underline{F} \underline{\varphi}, \underline{\psi})=(\underline{F} \underline{C} \underline{\varphi}, \underline{C} \underline{\varphi})=(\underline{C} \underline{F} \underline{\varphi}, \underline{C} \underline{\psi})=(\underline{\varphi}, \underline{F} \underline{\varphi}),
$$

from lemma 5. The other equalities follow from the self-adjointness of $F$ : Similarly for $\underline{G}$. This completes the proof.

Returning to $\Delta J$, setting

$$
\delta \underline{\varphi}=\delta \underline{u}+i \delta \underline{v},
$$


where $\underline{C} \delta \underline{u}=\delta \underline{u}, \underline{C} \delta \underline{v}=\delta \underline{v}$ (Iemma 7), we have$$
\underline{L} \delta \underline{\varphi}=(\underline{G}+i \underline{F})(\delta \underline{u}+i \delta \underline{v})=(\underline{G} \delta \underline{u}-\underline{F} \delta \underline{v})+i(\underline{F} \delta \underline{u}+\underline{G} \delta \underline{v}),
$$

$$
\left\{\begin{aligned}
(\underline{L} \delta \underline{\varphi}, \underline{C} \delta \underline{\varphi})= & (\{(\underline{G} \delta \underline{u}-\underline{F} \delta \underline{v})+i(\underline{F} \delta \underline{u}+\underline{G} \delta \underline{v})\}, \delta \underline{u}-i \delta \underline{v}) \\
= & \{(\underline{G} \delta \underline{u}-\underline{F} \delta \underline{v} ; \delta \underline{u})-(\underline{F} \delta \underline{u}+\underline{G} \delta \underline{v}, \delta \underline{v})\}+ \\
& +i\{(\underline{F} \delta \underline{u}+\underline{G} \delta \underline{v}, \delta \underline{u})+(\underline{G} \delta \underline{u}-\underline{F} \delta \underline{v}, \delta \underline{v})\} .
\end{aligned}\right.
$$

Now

Therefore,

$$
\left\{\begin{aligned}
\left(\delta \underline{\varphi}, \underline{\varphi}_{0}\right)^{2}-\left(\delta \underline{\varphi}, \underline{\psi}_{0}\right)^{2}= & \left\{\left(\delta \underline{u}, \underline{u}_{0}\right)^{2}+\left(\delta \underline{u}^{2}, \underline{v}_{0}\right)^{2}-\left(\delta \underline{v}, \underline{u}_{0}\right)^{2}-\left(\delta \underline{v}, \underline{v}_{0}\right)^{2}\right\} \\
& +2 i\left\{\left(\delta \underline{u}, \underline{u}_{0}\right)\left(\delta \underline{v}_{0} \underline{u}_{0}\right)+\left(\delta \underline{u}, \underline{v}_{0}\right)\left(\delta \underline{v}, \underline{v}_{0}\right)\right\}
\end{aligned}\right.
$$

Then,

$$
\begin{aligned}
& \left(\delta \underline{\varphi}, \underline{\varphi}_{0}\right)=\left(\delta \underline{u}+i \delta \underline{v}, \underline{u}_{0}\right)=\left(\delta \underline{u}, \underline{u}_{0}\right)+i\left(\delta \underline{v}, \underline{u}_{0}\right), \\
& \left(\delta \underline{\varphi}, \underline{\psi}_{0}\right)=\left(\delta \underline{u}+i \delta \underline{v}, i \underline{v}_{0}\right)=\left(\delta \underline{v}, \underline{v}_{0}\right)-i\left(\delta \underline{u}, \underline{v}_{0}\right) .
\end{aligned}
$$

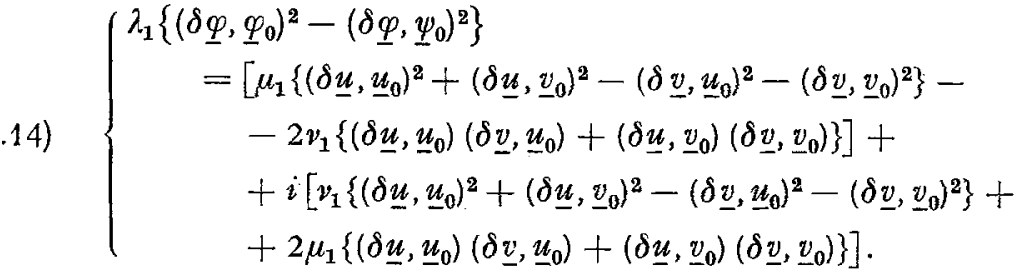

LEMMA 9. If $\underline{I}=\underline{P}+i \underline{Q}, \underline{P}$ and $\underline{Q}$ real, then $\Delta \underline{J}=\Delta \underline{P}+i \Delta \underline{Q}$, and

$$
\begin{aligned}
& \left\{\begin{aligned}
A \underline{P}= & 2\{(\underline{G} \delta \underline{u}, \delta \underline{u})-(\underline{G} \delta \underline{v}, \delta \underline{v})-2(\underline{F} \delta \underline{u}, \delta \underline{v})\}- \\
& -\mu_{1}\left\{\left(\delta \underline{u}^{\prime} \underline{u}_{0}\right)^{2}+\left(\delta \underline{u}, \underline{v}_{0}\right)^{2}-\left(\delta \underline{v}, \underline{u}_{0}\right)^{2}-\left(\delta \underline{v}, \underline{v}_{0}\right)^{2}\right\}+ \\
& +2 v_{1}\left\{\left(\delta \underline{u}, \underline{u}_{0}\right)\left(\delta \underline{v}, \underline{u}_{0}\right)+\left(\delta \underline{u}, \underline{v}_{0}\right)\left(\delta \underline{v}, \underline{v}_{0}\right)\right\} .
\end{aligned}\right. \\
& \left\{\begin{aligned}
\Delta \underline{Q}= & 2\{(\underline{F} \delta \underline{u}, \delta \underline{u})-(\underline{F} \delta \underline{v}, \delta \underline{v})+2(\underline{G} \delta \underline{u}, \delta \underline{v})\}- \\
& -v_{1}\left\{\left(\delta \underline{u}, \underline{u}_{0}\right)^{2}+\left(\delta \underline{u}, \underline{v}_{0}\right)^{2}-\left(\delta \underline{v}, \underline{u}_{0}\right)^{2}-\left(\delta \underline{v}, \underline{v}_{0}\right)^{2}\right\}- \\
& -2 \mu_{1}\left\{\left(\delta \underline{u}, \underline{u}_{0}\right)\left(\delta \underline{v}, \underline{u}_{0}\right)+\left(\delta \underline{u}_{v}, \underline{v}_{0}\right)\left(\delta \underline{v}^{2}, \underline{v}_{0}\right)\right\} .
\end{aligned}\right.
\end{aligned}
$$

Proof. (6.15) and (6.17) are obtained by combining (6.3), (6.10), and (6.14) and observing that all the inner products are real (lemma 5). The effect of lemma 9 is to express the increments of the real and imaginary parts of $\underline{J}$, as we move along the constraining surface, (5.12), away from a stationary point as real quadratic forms in $\delta \underline{u}$ and $\delta \underline{v}$. Since all the inner products which appear are real, we can think of the pair $\delta \underline{u}, \delta \underline{v}$ as being a point in the direct sum of two real Hilbert spaces. It should be observed however that these real Hilbert spaces are not spaces of real functions, but rather complicated spaces built up by using direct sums of complex function spaces and conjugation. 
LEMMA 10. Let $\underline{u}$ and $\underline{v}$ be obtained from the stationary point $\varphi(\alpha)$ (theorem 6 ) according to (6.4). Then

$$
\begin{aligned}
& \left(\underline{u}, \underline{u}_{0}\right)=\frac{\alpha+\bar{\alpha}}{2}\left[\frac{|\alpha|^{2}+1}{|\alpha|^{2}}\right] ; \quad\left(\underline{u}, \underline{v}_{0}\right)=-\frac{(\alpha-\bar{\alpha})}{2 i}\left[\frac{|\alpha|^{2}+1}{|\alpha|^{2}}\right], \\
& \left(\underline{v}, \underline{u}_{0}\right)=\frac{\alpha-\bar{\alpha}}{2 i}\left[\frac{|\alpha|^{2}-1}{|\alpha|^{2}}\right] ; \quad\left(\underline{v}, \underline{v}_{0}\right)=\frac{\alpha+\bar{\alpha}}{2}\left[\frac{|\alpha|^{2}-1}{|\alpha|^{2}}\right] .
\end{aligned}
$$

In general, if $\underline{\varphi}$ lies on (5.12), and $\underline{u}$ and $\underline{v}$ are given by (6.4), then

$$
\begin{aligned}
\left(\underline{u}, \underline{u}_{0}\right)^{2}+\left(\underline{u}, \underline{v}_{0}\right)^{2}-\left(\underline{v}, \underline{u}_{0}\right)^{2}-\left(\underline{v}, \underline{v}_{0}\right)^{2} & =4, \\
\left(\underline{u}, \underline{u}_{0}\right)\left(\underline{v}, \underline{u}_{0}\right)+\left(\underline{u}, \underline{v}_{0}\right)\left(\underline{v}, \underline{v}_{0}\right) & =0 .
\end{aligned}
$$

Proof. We shall carry out the computation for the first equation in (6.17).

$$
\begin{aligned}
\left(\underline{u}, \underline{u}_{0}\right) & =\frac{1}{2}\left(\underline{\varphi}(\alpha)+\underline{C} \underline{\varphi}(\alpha), \underline{\varphi}_{0}\right)=\frac{1}{2}\left(\varphi(\alpha), \underline{\varphi}_{0}\right)+\frac{1}{2}(\underline{\varphi}(\alpha)) \\
& =\frac{1}{2}\left(\left[\alpha \lambda_{1} \varphi_{1}, \frac{1}{\alpha} \lambda_{1} \psi_{1}\right],\left[\varphi_{0}, \bar{\varphi}_{0}\right]\right)+\frac{1}{2}\left(\left[\varphi_{0}, \bar{\varphi}_{0}\right],\left[\alpha \lambda_{1} \varphi_{1}, \frac{1}{\alpha} \lambda_{1} \psi_{1}\right]\right) \\
& =\frac{1}{2}\left\{\alpha+\frac{1}{\alpha}+\bar{\alpha}+\frac{1}{\alpha}\right\}=\frac{\alpha+\bar{\alpha}}{2}\left[\frac{|\alpha|^{2}+1}{|\alpha|^{2}}\right] .
\end{aligned}
$$

The remaining equations in (6.17) and (6.18) are derived in a similar manner. As for (6.19) and (6.20), they are obtained analogously to (6.13).

THEOREM 7. Let $\underline{u}$ and $\underline{v}$ be obtained by (6.4) from the stationary point $\underline{\varphi(\alpha)}$.

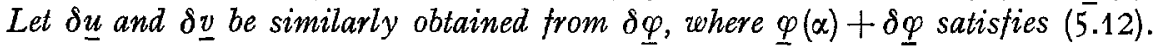
Then

(a) If $|\alpha| \neq 1$ there exists an $\varepsilon<0$, such that in the weak neighborhood of $\underline{\varphi}(\alpha)$ defined by $\left(\delta \underline{u}, \underline{u}_{0}\right)^{2}+\left(\delta \underline{u}, \underline{v}_{0}\right)^{2}<\varepsilon,\left(\delta \underline{v}, \underline{u}_{0}\right)^{2}+\left(\delta \underline{v}, \underline{v}_{0}\right)^{2}<\varepsilon$, the increment in the imaginary part of $\underline{J}$ satisfies

$$
\begin{aligned}
& \Delta Q]_{\delta \underline{u}=0}=-(\underline{F} \delta \underline{v}, \delta \underline{v}) \leqq 0, \\
& \Delta Q]_{\delta \underline{v}=0}=(\underline{F} \delta \underline{u}, \delta \underline{u}) \geqq 0 .
\end{aligned}
$$

(b) If $|\alpha|=1$, then $\Delta Q]_{\delta u=0}=-(\underline{F} \delta \underline{v}, \delta \underline{v}) \leq 0$.

Proof. Expanding (6.2) with the help of (6.13), we obtain $(6.23)\left\{\begin{aligned} 2\left\{\left(\underline{u}, \underline{u}_{0}\right)\left(\delta \underline{u}, \underline{u}_{0}\right)\right. & \left.\left.+\left(\underline{u}, \underline{v}_{0}\right)\left(\delta \underline{u}_{,} \underline{v}_{0}\right)-\left(\underline{v}, \underline{u}_{0}\right) \delta \underline{v}, \underline{u}_{0}\right)-\left(\underline{v}, \underline{v}_{0}\right)\left(\delta \underline{v}, \underline{v}_{0}\right)\right\}+ \\ & +\left(\delta \underline{u}, \underline{u}_{0}\right)^{2}+\left(\delta \underline{u}, \underline{v}_{0}\right)^{2}-\left(\delta \underline{v}, \underline{u}_{0}\right)^{2}-\left(\delta \underline{v}, \underline{v}_{0}\right)^{2}=0,\end{aligned}\right.$

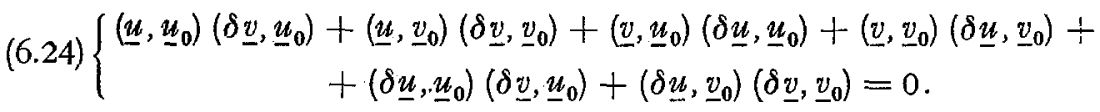

(a) Suppose $\delta \underline{u}=0$. Then $\delta \underline{v}$ must satisfy

$$
\begin{gathered}
2\left\{\left(\underline{v}, \underline{u}_{0}\right)\left(\delta \underline{v} \underline{u}_{0}\right)+\left(\underline{v}, \underline{v}_{0}\right)\left(\delta \underline{v}, \underline{v}_{0}\right)\right\}+\left(\delta \underline{v}^{\prime} \underline{u}_{0}\right)^{2}+\left(\delta \underline{v}, \underline{v}_{0}\right)^{2}=0 \\
\left(\underline{u}^{\prime} \underline{u}_{0}\right)\left(\delta \underline{v}, \underline{u}_{0}\right)+\left(\underline{u}, \underline{v}_{0}\right)\left(\delta \underline{v}, \underline{v}_{0}\right)=0 .
\end{gathered}
$$


From lemma 10, it is clear that not both $\left(\underline{u}, \underline{u}_{0}\right)=0$ and $\left(\underline{v}, \underline{v}_{0}\right)=0$. Therefore, from the homogeneity of $(6.20)$ and $(6.26)$, we have

$$
\left(\delta \underline{v}, \underline{u}_{0}\right)\left(\underline{v}, \underline{v}_{0}\right)-\left(\delta \underline{v}, \underline{v}_{0}\right)\left(\underline{v}, \underline{u}_{0}\right)=0
$$

But (6.27) and (6.25) imply by Cramer's Rule that either

$$
\left(\delta \underline{v}, \underline{u}_{0}\right)=\left(\delta \underline{v}, \underline{v}_{0}\right)=0
$$

or

$$
\left(\delta \underline{v}, \underline{u}_{0}\right)=-2\left(\underline{v}, \underline{u}_{0}\right) ; \quad\left(\delta \underline{v}, \underline{v}_{0}\right)=-2\left(\underline{v}, \underline{v}_{0}\right) .
$$

If (6.29) holds, then, from (6.18), we have

$$
\left(\delta \underline{v}, \underline{u}_{0}\right)^{2}+\left(\delta \underline{v}, \underline{v}_{0}\right)^{2}=4\left(|\alpha|-\frac{1}{|\alpha|}\right)^{2} .
$$

Let $\delta \underline{v}=0$. Then $\delta \underline{u}$ must satisfy

$(6.31) 2\left\{\left(\underline{u}, \underline{u}_{0}\right)\left(\delta \underline{u}_{,} \underline{u}_{0}\right)+\left(\underline{u}, \underline{v}_{0}\right)\left(\delta \underline{u}, \underline{v}_{0}\right)\right\}+\left(\delta \underline{u}, \underline{u}_{0}\right)^{2}+\left(\delta \underline{u}, \underline{v}_{0}\right)^{2}=0$,

$$
\left(\underline{v}, \underline{u}_{0}\right)\left(\delta \underline{u}, \underline{u}_{0}\right)+\left(\underline{v}, \underline{v}_{0}\right)\left(\delta \underline{u}, \underline{v}_{0}\right)=0 \text {. }
$$

Since it follows from $(6.18)$ that not both $\left(\underline{v}, \underline{u}_{0}\right)=0$ and $\left(\underline{v}, \underline{v}_{0}\right)=0$, we find that here $\delta \underline{u}$ must satisfy either

$$
\left(\delta \underline{u}, \underline{u}_{0}\right)=\left(\delta \underline{u}, \underline{v}_{0}\right)=0
$$

or

$$
\left(\delta \underline{u}, \underline{u}_{0}\right)^{2}+\left(\delta \underline{u}, \underline{v}_{0}\right)^{2}=4\left(|\alpha|+\frac{1}{|\alpha|}\right)^{2} .
$$

If we let $\varepsilon \leq 4\left(|\alpha|-\frac{1}{|\alpha|}\right)^{2}$, then in the neighborhood determined by this $\varepsilon$, when $\delta \underline{u}=0(6.30)$ is excluded, and when $\delta \underline{v}=0,(6.34)$ is excluded. Therefore, from (6.16), (6.28), and (6.33) in this neighborhood (6.21) and (6.22) are valid.

(b) $|\alpha|=1$. The discussion above applies through (6.30). But if $|\alpha|=1$, both $(6.28)$ and $(6.30)$ imply $\left(\delta \underline{v}, \underline{v}_{0}\right)=\left(\delta \underline{v}, \underline{u}_{0}\right)=0$, so the theorem is proved. We can now interpret theorem 7 in terms of the principle formulated in $\S 4$.

COROLLARY. Let $\varphi_{s}=\alpha \lambda_{1} \varphi_{1}, \psi_{s}=\frac{1}{\alpha} \lambda_{1} \psi_{1}$ be a pair of functions which make $J=(A \varphi, \underline{\psi})$ stationary subject to the constraint $\left(\varphi, \varphi_{0}\right)\left(\varphi_{0}, \bar{\psi}\right)=1$. Then if $\varphi$ and $\psi$ are varied about $\varphi_{s}, \psi_{s}$ subject to the constraint, then

(a) if $|\alpha| \neq 1$, and if $\varphi+\bar{\psi}$ is held constant, then $\operatorname{Im} J$ will have a local maximum at $\varphi_{s}, \psi_{s}$. If $\varphi-\bar{\psi}$ is held constant, Im $J$ will have a local minimum at $\varphi_{s}, \psi_{s}$.

(b) if $|\alpha|=1$, and if $\varphi+\bar{\psi}$ is held constant, then $\operatorname{Im} J$ will have a global maximum.

\section{§7. The variational principle for the reflection amplitude}

The complex reflection amplitude in one dimensional quantum scattering is given by

$$
\frac{1}{\mu_{1}} \stackrel{\infty}{=} \int_{-\infty}^{\infty} \omega(x) V(x) e^{+i k x} d x
$$


where $\omega(x)$ is the solution of the integral equation (3.4). If, as before $\varphi_{0}$ is defined by (2.4), the appropriate integral equations corresponding to the set (2.8) are

$$
A \varphi=\varphi_{0}, \quad A^{*} \bar{\psi}=\bar{\varphi}_{0} .
$$

As noted in the proof of theorem 1, these equations have a unique solution in $H$. Moreover in view of lemma 1 , here $\bar{\psi}=\bar{\varphi}$. The reciprocity relation corresponding to (2.9) reduces here to the trivial statement that

$$
\left(\varphi_{0}, \bar{\varphi}\right)=\left(\varphi, \bar{\varphi}_{0}\right) .
$$

Now if $\varphi_{1}$ once again is the unique solution to the first of the set (7.2), then

$$
\frac{1}{\mu_{1}}=\left(\varphi_{1}, \bar{\varphi}_{0}\right)
$$

so that here we may treat (7.2) instead of (3.4).

The variational principle for the determination of $1 / \mu_{1}$ consists in this case of making

$$
J^{R}=(A \varphi, \bar{\varphi})
$$

stationary subject to the constraint

$$
\left(\varphi, \bar{\varphi}_{0}\right)=1 \text {. }
$$

To accomplish this, we introduce the Lagrange multiplier $\mu$, and set

$$
J_{\mu}^{R}=J^{R}-2 \mu\left(\varphi, \bar{\varphi}_{0}\right)
$$

and obtain the result that $J^{R}$ is stationary if and only if

$$
A \varphi=\mu \varphi_{0} .
$$

This last equation corresponds to the set (4.5). Here, as in the set (7.2), the second equation is merely the conjugate of this and consequently it may be omitted. Equation (7.4) is simpler in appearance than the set (4.5) since here we may use a linear rather than a quadratic constraint. Actually, if we think of the variational principles (5.11), (5.12), or (5.13) abstractly; that is, independent of the interpretation given to them in $\S 5,(7.3)$ and (7.4) can be thought of as special cases of (5.11) and (5.12) obtained by replacing $\varphi_{0}$ by $C \varphi_{0}$, setting $\psi_{0}$ equal to zero and interpreting $C$ as usual conjugation. While a more unified treatment would result if (5.13) were similarly specialized, the simplifications resulting from the use of a linear rather than a quadratic restraint make the use of (7.4) preferable.

In order to obtain a theorem analogous to theorem 3, we now make the additional assumption that $\left(\varphi_{1}, \bar{\varphi}_{0}\right) \neq 0$. Physically this assumption is of course equivalent to assuming that the potential barrier is at least partially reflecting. It is moreover, not equivalent to assuming that the potential barrier is not transparent [i.e., that $\left.\left(\varphi_{1}, \varphi_{0}\right) \neq 0\right]$. It is interesting to note 
that the existence of partial reflection implies the existence of partial transmission. More explicitly we have the following:

LEMMA 11. Let $\varphi_{1}$ be the unique solution to (7.2). Then $\left(\varphi_{1}, \bar{\varphi}_{0}\right) \neq 0$ implies that $\left(\varphi_{1}, \varphi_{0}\right) \neq 0$.

Proof. The argument immediately below equation (3.15) shows that $\left(\varphi_{1}, \varphi_{0}\right)=0$ implies $\left(\varphi_{1}, \bar{\varphi}_{0}\right)=0$. The lemma follows by logical equivalence.

THEOREM 8. There exists exactly one value of $\mu, \mu_{1}$, such that $J^{R}$ can be made stationary. $\mu_{1}$ is equal to the value of $J^{R}$ when $J^{R}$ is made. stationary subject to the constraint (7.4). Simultaneously,

$$
\mu_{1}=\frac{1}{\left(\varphi_{1}, \bar{\varphi}_{0}\right)} .
$$

Since the proof is nearly identical to that of theorem 3, we will content ourselves with remarking that if $\varphi_{s}$ satisfies (7.5) it must be of the form $\varphi_{s}=\mu \varphi_{1}$, since $\varphi_{1}$ is the unique solution of (7.2). Moreover since $\varphi_{s}$ must also satisfy (7.4), it follows that $\mu\left(\varphi_{1}, \bar{\varphi}_{0}\right)=1$. Now (7.5) implies that

$$
(A \varphi, \bar{\varphi})=\mu\left(\varphi_{0}, \bar{\varphi}\right)=\mu \text {. }
$$

Finally, in analogy to the corollary of theorem 7 , we have

THEOREM 9. Let $\varphi_{s}=\mu \varphi_{1}$ be any function which makes $J^{R}=(A \varphi, \bar{\varphi})$ stationary subject to the constraint $\left(\varphi, \bar{\varphi}_{0}\right)=1$, then

(a) if $\varphi+\bar{\varphi}$ is held constant, $\operatorname{Im} J^{R}$ will have a global minimum at $\varphi_{s}$;

(b) if $\varphi-\bar{\varphi}$ is held constant, Im $J^{R}$ will have a global maximum at $\varphi_{s}$;

Proof. Since here we are dealing with the linear constraint (7.4), the second variations of $J^{R}$ and $J_{u}^{R}$ are identical for any function $\varphi_{s}$ which makes them stationary. The nature of the stationary point is therefore determined completely by the second variation $\delta^{2} J^{R}$ which is

$$
\delta^{2} J^{R}=(A \delta \varphi, \delta \bar{\varphi}) .
$$

From this we find from (2.3),

$$
\begin{aligned}
2 i \operatorname{Im} \delta^{2} J^{R} & =(A \delta \varphi, \delta \bar{\varphi})-\overline{(A \delta \varphi, \delta \bar{\varphi})} \\
& =(A \delta \varphi, \delta \bar{\varphi})-\left(A^{*} \delta \bar{\varphi}, \delta \varphi\right) \\
& =(\delta \varphi, \delta \bar{\varphi})-(\delta \bar{\varphi}, \delta \varphi)+(K \delta \varphi, \delta \bar{\varphi})-\left(K^{*} \delta \bar{\varphi}, \delta \varphi\right) .
\end{aligned}
$$

Letting $\varphi=u+i v$ where $u$ and $v$ are real so that

$$
\delta \varphi=\delta u+i \delta v
$$

and using (2.6) we find that

$$
2 \operatorname{Im} \delta^{2} J^{R}=2(\delta u, \delta v)+(F \delta u, \delta u)-(F \delta v, \delta v)+2(G \delta u, \delta v) .
$$

The theorem then follows from lemma 3 since $F$ is negative semi-defir ite.

In conclusion it should be remarked that we have been unable to find useful analog of theorem 5 for this case. 


\section{References}

[1] DoLpH, C. L.: The Mathematician Grapples with Linear Problems Associated with the Radiation Condition. Trans. Inst. Radio Engineers PGAP, AP-4, (1956). - [2] DuNFord, N.: Spectral operators. Pac. J. Math. 4, 321--354 (1954). — [3] Hille, E.: Functional Analysis and Semi-Groups. Amer. Math. Soc., Colloquium Publ. 31 (1948). [4] Jones, D. S.: A Critique of the Variational Method. Trans. Inst. Radio Engineers PGAP, AP-4, (1956). - [5] Kato, T.: Note on Schwinger Variational Principle. Progress of Theoretical Physics (Japan) 6, 295-305 (1951). - - [6] Marcuvirz, N.: Recent Developments in Wave Propagation, Notes, Part III-D. New York University 1953. [7] Morse and FeshBach: Methods of Theoretical Physics, Vol II, Chapters 9, 11. New York: McGraw Hill 1953. - [8] RIEsz, F., and B. Sz. NAGY: Lecons d'Analyse Fonctionnelle. Budapest 1952. - [9] Schwinger, J., and H. Levine: On the Theory of Diffraction by an Aperture in an Infinite Plane Screen. I. Physical Review 74, $958-974$ (1948).

Engineering Res. Inst., University of Michigan, Ann Arbor, Mich. (U.S.A.)

(Eingegangen am 9. Januar 1956) 